\title{
Validación del cuestionario de auto-reporte de comportamiento antisocial en adolescentes: CACSA $^{1}$
}

\author{
Paula Alarcón Bañares ${ }^{2}$ \\ Ricardo Pérez-Luco \\ Sonia Salvo \\ Gloria Roa \\ Katherine Jaramillo \\ Carla Sanhueza \\ Universidad de La Frontera, Temuco, Chile
}

\begin{abstract}
Resumen: El estudio tiene por objetivo presentar la validación de un instrumento formulado racionalmente para evaluar, mediante auto-reporte, el comportamiento social en adolescentes chilenos (CACSA) y describir el comportamiento social de dos grupos: adolescentes escolarizados $(\mathrm{N}=1914)$ y adolescentes infractores de ley $(\mathrm{N}=264)$. El cuestionario posee 56 ítemes que exploran tres dimensiones generales, a) Comportamiento Prosocial (CPRO), b) Comportamiento Antisocial Total (CAT); y c) Víctima de Abuso de Poder (VAP); CAT contiene seis dimensiones específicas: Delitos (D), Transgresiones Contra la Propiedad (TPRO), Transgresiones Contra las Personas (TPER), Comportamiento Abusivo con Pares (CAP), Consumo de Alcohol y Drogas (OHD); y Transgresiones Sociales Leves (TSL). El estudio incluye también una escala de deseabilidad social y otra actitudinal. Los resultados revelan importantes fortalezas psicométricas del instrumento y capacidad de discriminación entre adolescentes escolarizados e infractores de ley. Se discute la importancia de reconocer por auto-reporte la prevalencia de comportamientos antisociales y la relación entre conductas prosociales y antisociales en adolescentes chilenos.
\end{abstract}

Palabras clave: adolescencia, comportamiento social, auto-relato.

\section{Validação de questionário de auto-relato de comportamento anti-social em adolescentes: CACSA}

Resumo: O estudo tem por objetivos apresentar a validação de um instrumento formulado racionalmente para avaliar, mediante auto-relato, o comportamento social de adolescentes chilenos (CACSA) e descrever o comportamento social de dois grupos: adolescentes escolarizados $(\mathrm{N}=1914)$ e adolescentes infratores da lei $(\mathrm{N}=264)$. O questionário consiste de 56 itens que exploram três dimensões gerais: a) Comportamento Pró-social (CPRO), b) Anti-Social (CAT) c) Vítimas de Abuso de Poder (VAP). CAT contém seis dimensões específicas: Delitos (D), Transgressões de Ofensa à Propriedade (TPRO), Transgressões Contra as Pessoas (TPER), Comportamentos Abusivos entre Pares (CAP), Consumo do Álcool e Drogas (OHD) e Transgressões Sociais Leves (TSL). O estudo incluiu também uma escala de desejabilidade social e outra atitudinal. Os resultados revelam que o instrumento mostra excelentes qualidades psicométricas e capacidade de discriminação entre os adolescentes escolarizados e os infratores da lei. Discute-se a importância de reconhecer a prevalência de comportamentos anti-sociais por meio de auto-relato e a relação entre condutas pró-sociais e anti-sociais em adolescentes chilenos.

Palavras-chave: adolescência, comportamento social, auto-relato.

\section{Validation of a self-report questionnaire addressing antisocial behavior in adolescents: CACSA}

\begin{abstract}
This paper reports the validation of a rationally built self-report instrument to assess social behavior in Chilean adolescents (CACSA) and presents the findings on social behavior from two samples: school attending adolescents $(\mathrm{N}=1914)$ and juvenile offenders $(\mathrm{N}=264)$. The questionnaire consists of 56 items, which explore three general behavioral dimensions: Prosocial (CPRO) and Antisocial (CAT) behavior, and Victims of Abuse of Power (VAP); CAT contains six specific dimensions: Crimes (D), Transgressions Against Property (TPRO), Transgressions Against Persons (TPER); Abusive Behavior with Peers (CAP), Substance Abuse (OHD); and Minor Social Transgressions (TSL). The study also includes a social desirability scale and an attitudinal scale. Results revealed the questionnaire has important psychometric strengths, as well as discriminative properties between the school attending adolescents and the juvenile offenders. The importance and reliability of self-report data for building indices of antisocial behavior is discussed, as well as the relationship between prosocial and antisocial behavior among Chilean adolescents.
\end{abstract}

Keywords: adolescence, antisocial behavior, self report.

\footnotetext{
${ }^{1}$ Esta investigación recibió financiamiento del Fondo Nacional de Desarrollo Científico y Tecnológico de Chile FONDECYT, a través del Proyecto 1070397.

${ }^{2}$ Endereço para correspondência:

Paula Alarcón Bañares. Universidad de La Frontera. Facultad de Educación y Humanidades. Departamento de Psicología. Av. Francisco Salazar 01145, Temuco, Chile.E-mail: paulandr@ufro.cl
}

Los patrones de comportamiento social en adolescentes durante los últimos años han sido descritos en diversas investigaciones, reportando interesantes cambios en la expresión tanto de comportamientos prosociales como antisociales. La mayoría de las investigaciones, caracterizan el comportamiento adolescente con un aumento progresivo 
de comportamientos antisociales desde contextos escolares (bullying), vandalismo en lugares públicos, hasta conductas delictivas con un mayor compromiso de violencia; pero paradojalmente, se observa también a los adolescentes, como los principales protagonistas de actos altruistas y prosociales (organizaciones estudiantiles, scouts, ecologistas, movimientos por la paz).

El comportamiento social de los adolescentes no puede ser estudiado de manera aislada, de los contextos en los que se desarrollan, ni de las variables evolutivas y culturales que subyacen a estas manifestaciones. La complejidad implica reconocer los puntos de tensión e integración que surgen en el proceso de adaptación social, pues los adolescentes contemporáneos, de diversos estratos sociales, están expuestos a una mayor concentración de cambios y transformaciones en su vida psicológica, lo que agudiza la crisis de identidad a la vez de dotarles de mayor autonomía y poder. Maluf (2002) describe la sociedad contemporánea como sociedad del riesgo, por la presencia de una escala de valores en permanente transformación, crisis de las certezas y de los modelos familiares y sobrevaloración del consumo y de los bienes económicos, lo que expone a los adolescentes a múltiples $\mathrm{y}$ urgentes presiones que pueden elevar los niveles de incertidumbre y estrés para algunos y/o generar mayor empoderamiento en otros, transformando sus conductas sociales y trayectorias evolutivas. Así también se ha descrito en estas sociedades urbanas modernas que la desintegración social y disolución de redes de apoyo en grupos sociales primarios es una variable clave que se asocia a la génesis de problemas psicosociales como la transgresión social y delincuencia (Gracia \& Herrero, 2006). Chile, por sus transformaciones sociopolíticas y económicas y por el desarrollo de las telecomunicaciones e informática, se ha transformado en un país de acelerada modernización en Latinoamérica, lo que impacta en los contextos sociales que moldean a los adolescentes, mostrando un incremento desde los años 1986 y 2002 en un $398 \%$ en las detenciones realizadas por carabineros (policía) en el tramo de 16 a 17 años (Werth \& Sepúlveda, 2003).

Según afirman investigadores clásicos en conducta antisocial y delincuencia juvenil como Farrington (1996) y Moffitt (1993), los primeros años de la adolescencia aparecen como periodo crítico para transgredir. Se ha observado que las tasas más elevadas de transgresión social se manifiestan a esta edad, en torno a los 15 años, y disminuyen paulatinamente (Ortega Ruiz \& Del Rey Alamillo, 2005), siendo así la etapa adolescente el período de mayor prevalencia de conductas antisociales, aunque son las transgresiones leves las conductas más frecuentes en esta etapa (Serrano, El Astal, \& Faro, 2004) y se concentran mayoritariamente en los grupos de adolescentes varones, mostrando las adolescentes mujeres un mayor predominio de comportamientos pro-sociales en la adolescencia (Inglés e cols., 2009). Un estudio reciente en Canadá a través de la técnica de auto-reporte, demuestra que más del 95\% de los adolescentes hombres y el 65\% de las adolescentes mujeres, confiesa haber cometido un acto delictual en el curso de los últimos doce meses (Dionne \& Cournoyer, 2006). En Chile, Hein y Barrientos (2004) coinciden con los estudios internacionales, mostrando que las conductas transgresoras auto-reportadas por adolescentes de la región metropolitana presentan niveles significativamente más elevados que las cifras judiciales registradas en faltas y actos delictivos. Los estudios de auto-reporte en comportamientos sociales y antisociales, ampliamente difundidos por investigaciones internacionales (Mestre, Samper, Nacher, Tur, \& Cortés, 2006; Tur, Mestre, \& Barrio, 2004) son escasos en población latinoamericana, desatendiendo un campo importante para el desarrollo de estrategias de prevención y detección precoz de comportamientos de riesgo en nuestros países. Lo señalado fundamenta la necesidad de contar con instrumentos de auto-reporte, no sólo adaptados sino desarrollados localmente y sensibles a nuestras especificidades sociales y culturales.

Respondiendo a la necesidad señalada, a continuación se expone el proceso de validación de un instrumento de auto-reporte para evaluar comportamiento antisocial y a su vez pro-social estudiando sus propiedades psicométricas a través de dos muestras, una de adolescentes secundarios y otra de adolescentes infractores de ley. Un segundo objetivo permitió caracterizar el comportamiento social auto-reportado por los jóvenes participantes del estudio, a través del instrumento ya validado.

El Cuestionario de Auto-reporte de Comportamiento Social Adolescente - CACSA - es un nuevo instrumento que busca detectar comportamiento social adaptativo y transgresor en adolescentes chilenos, permitiendo una aproximación descriptiva y comprensiva a las manifestaciones que ellos normalizan en su convivencia social, con especial atención en las dimensiones de lo prosocial y antisocial según género y tramo de edad.

\section{Marco conceptual}

Se iniciará la revisión conceptual con el estudio del comportamiento prosocial definido como todo comportamiento voluntario orientado al beneficio de otros, incluyendo procesos cognitivos como el razonamiento prosocial y procesos emocionales como la empatía (Calvo, González, \& Martorell, 2001; Mestre Escrivá, Samper García, \& Frías Navarro, 2002; Mestre, Tur, Samper, Nacher, \& Cortés, 2007); y que se asocia a mayor asertividad, cooperativismo y popularidad en adolescentes escolarizados (Inglés e cols., 2009). Las investigaciones desde la perspectiva evolutiva han demostrado que el comportamiento prosocial se torna estable en los primeros años de la adolescencia (Caprara, Steca, Zelli, \& Capanna, 2005) y actúa como factor de protección de la desadaptación, pues las conductas prosociales muestran una relación inversamente proporcional al comportamiento antisocial, previniendo tal comportamiento (López Latorre, Garrido Genovés, Rodríguez Díaz, \& Paíno Quesada, 2002). Mestre y cols. (2002) señalan que la empatía con el 
sufrimiento de los demás es clave para limitar la agresión a otros, dos de sus principales componentes, preocupación empática (sentimientos de tristeza y preocupación ante la necesidad de otra persona) y toma de perspectiva (habilidad para comprender el punto de vista del otro) permiten a los adolescentes ser menos agresivos por su sensibilidad emocional y capacidad para comprender las consecuencias negativas de su actos. Al respecto, se ha constatado diferencias de género en la conducta prosocial, observándose que las mujeres muestran mayor predisposición empática y menos comportamientos agresivos y transgresores que los hombres durante la adolescencia (Garaigordobil, Maganto, Perez, \& Sansinenea, 2009). Asimismo, estudios transculturales describen una interesante variabilidad en el comportamiento social, observándose puntuaciones más elevadas en comportamiento prosocial de adolescentes españoles al compararlos con adolescentes norteamericanos, peruanos, argentinos e italianos (González, Martorell, Casulla, \& Calvo, 1998; Marzocchi e cols., 2004; Roa, Del Barrio, \& Carrasco, 2004), sin embargo, las expresiones antisociales en esta misma población también aparecen más elevadas al contrastarlas con muestras de adolescentes portugueses, palestinos y peruanos (Roa e cols., 2004; Serrano e cols., 2004). Las variables culturales se manifiestan en los comportamientos adolescentes en la dirección prosocial y antisocial, siendo de especial relevancia la vertiente de transgresión que según estudios comparativos coinciden en reflejar el aumento progresivo en diferentes países.

El comportamiento transgresor y antisocial adolescente es un fenómeno que ha desafiado a múltiples investigadores en las últimas décadas y cuenta con una amplia revisión desde diversos paradigmas, jurídico, clínico-psicopatológico, sociológico y con acertados alcances en corrientes criminológicas integradoras, donde estudios de seguimiento incorporan la perspectiva evolutiva y empírica para describir las trayectorias de desadaptación adolescente (Farrington, 1996; Fréchette \& Le Blanc, 1998).

Este estudio se adscribe a las teorías del contextualismo evolutivo o "life course" para la definición de desadaptación social, comprendiendo el fenómeno como un proceso evolutivo, donde los comportamientos se desvían de las normas sociales para incurrir en la transgresión de reglas o límites previamente establecidos por la cultura y organización social imperante. Estos comportamientos transgresores pueden presentarse, antes o durante la adolescencia, en forma aislada o bien de modo reiterado, perpetuándose en algunos casos un estilo conductual antisocial delictivo; por tanto, resulta relevante distinguir entre un comportamiento transgresor y lo propiamente delictivo, ya que algunos comportamientos descritos como transgresores están dentro del margen de lo esperable en entornos adolescentes, en contraste con la conducta antisocial y/o delictiva que ha derivado en procesos o modos habituales de desadaptación social, en los que confluyen además de la adolescencia, factores de riesgo personal, familiar, social y cultural.
Los estudios criminológicos han demostrado que se detecta un tercio del comportamiento delictivo, siendo la mayoría de las transgresiones invisibles al control judicial en diferentes países del mundo (Garrido, Stangeland, \& Redondo, 1999). Estudiar la historia de vida de adolescentes infractores ha permitido identificar la importancia de factores de riesgo asociados a la persistencia en las carreras delictivas. Así, los metanálisis resaltan el inicio precoz, relación con pares con problemas delictuales, escasa supervisión de los padres y abuso de drogas, como los principales factores de riesgo para que un adolescente se inicie y mantenga en una trayectoria antisocial (Fréchette \& Le Blanc, 1998). Desde esta mirada, los estudios de auto-reporte se transforman en una metodología que permitan hacer visible transgresiones iniciales o manifestaciones desadaptativas que no son detectadas en períodos críticos de mayor receptividad a las intervenciones.

Otro tipo de análisis de comportamientos antisociales en adolescentes, emergente en Chile, es el estudio de violencia escolar o abuso de poder entre pares, particularmente a través del estudio del fenómeno bullying o matonaje, descrito como toda conducta de persecución física y/o psicológica que realiza un estudiante contra otro, al que escoge como víctima de repetidos ataques.

Definido inicialmente por Olweus (1993), su comprensión aún está pendiente. Estadísticas recientes en Chile muestran que un $54 \%$ de los adolescentes varones y $36 \%$ de mujeres auto-reportan haber ejercido el bullying (Consejo Nacional para el Control de Estupefacientes [CONACE], 2003) junto a un $31 \%$ que reporta ser víctima reiterada de acoso de sus pares (UNICEF, 2004). Otra investigación en la Región Metropolitana demostró que el 14\% de los encuestados percibía que en sus establecimientos educacionales existía bastante o mucha violencia, el 17\% indicó que había sido agredido físicamente en el último año, y el 48\% insultado o agredido verbalmente en el mismo periodo de tiempo (García \& Madriaza, 2005). Los datos demuestran que no hay diferencias en los niveles de violencia de acuerdo a nivel socioeconómico de los estudiantes, al igual que lo señalado en una encuesta de auto-reporte de comportamientos de riesgo de Hein y Barrientos (2004). Esta información permite desmitificar la conducta antisocial adolescente vinculada a contextos de riesgo social y económico, haciendo visible el fenómeno en todos los grupos sociales.

El auto-reporte a nivel internacional de comportamientos antisociales, ha sido la técnica que ha permitido demostrar que las conductas antisociales tienen su mayor prevalencia en la adolescencia, que los comportamientos se distribuyen de modo homogéneo en diferentes grupos o clases sociales, y que existen variaciones culturales y de género en su manifestación. Las mujeres aparecen transgrediendo menos que los varones en relaciones $4: 1$ ó 3:1, a pesar de estudios recientes que describen una mayor participación de las jóvenes en actos delictivos y cifras similares en consumo de alcohol y drogas (CONACE, 2007). 
La técnica de auto-reporte permite estudiar frecuencia e intensidad de los comportamientos desadaptados, superando las limitaciones de las estadísticas oficiales o la sobrerepresentación que le dan los medios de comunicación al problema. Los datos de auto-reporte muestran que hasta el 90\% de los adolescentes reconocen haberse implicado en al menos una actividad antisocial antes de alcanzar los 18 años de edad; esta información puede resultar alarmante al compararla con los archivos oficiales que muestran índices de delincuencia mucho menores: $15 \%$ a $35 \%$ en varones y $2 \%$ a $14 \%$ en mujeres (Rutter \& Giller, 1985). En cuanto a confiabilidad de la técnica de auto-reporte Huizinga y Elliott (1986) encontraron alta asociación en los estudios que comparan cifras oficiales con auto-informes de personas transgresoras y procesadas por el sistema judicial (Rutter, Giller, \& Hagell, 2000).

Existe un vasta variabilidad de instrumentos de auto-reporte en comportamientos sociales ampliamente difundidos en población española y latinoamericana (Bringas, Herrero, Cuesta, \& Rodríguez, 2006; Martorell \& Gonzalez, 1992; Seisdedos, 1988), pero destaca de modo especial el estudio de Cambridge sobre delincuencia auto-informada en el Reino Unido, Europa y Canadá (Farrington e cols., 2003), pues usando este método permitió dimensionar a nivel internacional el comportamiento antisocial juvenil invisibilizado por el sistema (Junge-Tas, Terlouw, \& Klein, 1994). La evidencia empírica acumulada apoya que el sesgo por falta de sinceridad y memoria, es mínimo como método de investigación para las poblaciones estudiadas; la mayor dificultad se concentra en la validez externa, sin embargo, en estudios donde se contempla registros oficiales, los jóvenes con mayor registro de delitos oficiales, en los auto-informes aparecen también con las cifras más altas (Rutter y cols., 2000).

En Chile se han publicado escasas investigaciones en esta línea, Hein y Barrientos (2004), usando instrumentos adaptados a realidad nacional, reportan, para tres regiones del país, un 34,4\% de conductas de riesgo antisocial y sólo un 3,3\% que reporta alta frecuencia y diversidad. El comportamiento de daños a la propiedad y robo muestra la más alta frecuencia con un 18,2\% y 14,2\% respectivamente, y en daños a personas la conducta más frecuente fue peleas de pandilla con un $10,9 \%$. Se observó un aumento progresivo por edad y con una mayor prevalencia en varones. Como se señaló anteriormente los datos muestran alta coincidencia con la evidencia empírica demostrada internacionalmente.

En síntesis, los contextos culturales, sociopolíticos y generacionales son variables que se suman a la multidimensionalidad como factores de riesgo, protección o mediatización de las expresiones prosociales y antisociales de los adolescentes, siendo importante para una comunidad registrar y detectar precozmente estas manifestaciones, a fin de responder con políticas públicas preventivas y medidas de control social apropiadas. Un ejemplo en Chile, es la rebaja en la edad de responsabilidad penal adolescente (a 14 años) a través de la LRPA No 20.084 y las medidas de sanción y privación de libertad implementadas.
Este estudio tiene por objetivo presentar la validación del CACSA, un instrumento racionalmente construido para evaluar mediante auto-reporte el comportamiento social de adolescentes chilenos; describiendo el comportamiento antisocial y pro-social de un grupo de adolescentes escolarizados y otro de adolescentes infractores de ley.

La relevancia de esta investigación radica en validar un instrumento de auto-reporte creado en Chile y determinar prevalencia y frecuencia de los comportamientos prosociales $\mathrm{y}$ antisociales en una amplia población de adolescentes.

\section{Método}

\section{Participantes}

Para el presente estudio se contó con una muestra total de 2.178 adolescentes, distribuidos en dos muestra: M1 estudiantes $(\mathrm{N}=1914)$, y M2 infractores $(\mathrm{N}=264)$, todos los cuales respondieron adecuadamente el CACSA. El 12,1\% de los estudiantes secundarios encuestados son adolescentes sancionados por algún delito y el 78,9\% restante son adolescentes que asisten regularmente a estudios secundarios, de los cuales el $47 \%$ son hombres y el 53\% mujeres. El 44\% de la muestra procede de la Región de Bío Bío, un 25\% de La Araucanía, un 16\% de Los Ríos; y un 15\% de Los Lagos, lo cual es proporcionalmente representativo de la población de las cuatro regiones estudiadas. La distribución de la muestra de estudiantes es relativamente equivalente por nivel de estudios, con leve predominio de $2^{\circ}$ Medio (16 años) que alcanza un $29 \%$ del total y leve disminución de $4^{\circ}$ Medio (18 años) que representa un 20\%; en tanto la muestra de infractores se divide en un 55\% sancionado con medidas en medio libre y un $45 \%$ con privación de libertad. Por último, el ingreso económico auto-reportado se distribuye de modo similar al de la población, reflejado también en la distribución en base a dependencia de los establecimientos educativos, la muestra de establecimientos municipalizados y subvencionados alcanzó un $68,5 \%$ y la muestra de particulares pagados a los que acceden las familias de mayores ingresos representó un 31,5\% de la muestra total; por su parte los ingresos reportados por los adolescentes infractores revelan que un 91,3\% pertenece al mundo de la pobreza.

La muestra de adolescentes escolarizados es estadísticamente representativa de la población de estudiantes secundarios de las cuatro regiones estudiadas; lo que se determina mediante muestreo estratificado por conglomerados con un $3 \%$ de error muestral estimado según el método de Scheaffer, Mendenhall y Ott (1987), considerando como estrato el nivel de estudio $-1^{\circ}$ a $4^{\circ}$ medio - y conglomerado a los establecimientos educacionales específicos según su dependencia administrativa (privados, subvencionados y municipales). Se extrae una submuestra de adolescentes que en el instrumento en estudio manifiestan un alto nivel de comportamiento antisocial $(N=123)$.

La muestra de adolescentes infractores de ley Muestra 2 ( $N=264$, sólo varones) está constituida por adolescentes 
sancionados por infracciones a la ley penal en las modalidades de privación de libertad y medio libre (ver Tabla 1), definiéndose como fenoménicamente representativa. Esta muestra se segmenta en adolescentes infractores persistentes con más de 3 delitos oficiales $(N=94)$, adolescentes con inicio de escalada delictiva, un solo delito oficial $(N=95)$, e infractores en transición delictiva, con un delito oficial, pero más antecedentes no oficiales $(N=75)$.

Tabla 1

Caracterización descriptiva de las muestras de estudio

\begin{tabular}{|c|c|c|c|c|c|c|c|c|c|c|}
\hline & \multicolumn{2}{|c|}{ Bío-Bío } & \multicolumn{2}{|c|}{ Araucanía } & \multicolumn{2}{|c|}{ Los Ríos } & \multicolumn{2}{|c|}{ Los Lagos } & \multicolumn{2}{|c|}{ Total } \\
\hline & f & $\%$ & f & $\%$ & f & $\%$ & f & $\%$ & f & $\%$ \\
\hline Población Escolar & 64.789 & $48 \%$ & 32.609 & $24 \%$ & 13.051 & $10 \%$ & 24.237 & $18 \%$ & 134.686 & $100 \%$ \\
\hline \multicolumn{11}{|l|}{ Muestra Estudiantes } \\
\hline Estimada & 530 & $48 \%$ & 267 & $24 \%$ & 107 & $10 \%$ & 198 & $18 \%$ & 1.102 & $100 \%$ \\
\hline Obtenida & 915 & $48 \%$ & 426 & $22 \%$ & 300 & $16 \%$ & 273 & $14 \%$ & 1.914 & $100 \%$ \\
\hline Diferencia & 385 & $73 \%$ & 159 & $60 \%$ & 193 & $180 \%$ & 75 & $38 \%$ & 812 & $74 \%$ \\
\hline \multicolumn{11}{|l|}{ Muestra Infractores } \\
\hline Medio Libre & 17 & $43 \%$ & 62 & $52 \%$ & 28 & $55 \%$ & 39 & $74 \%$ & 146 & $55 \%$ \\
\hline Privación de Libertad & 23 & $58 \%$ & 58 & $48 \%$ & 23 & $45 \%$ & 14 & $26 \%$ & 118 & $45 \%$ \\
\hline Total & 40 & $15 \%$ & 120 & $45 \%$ & 51 & $19 \%$ & 53 & $20 \%$ & 264 & $100 \%$ \\
\hline
\end{tabular}

En este estudio a) se estudian las propiedades psicométricas del CACSA y b) se describe y caracteriza a dos muestras de adolescentes chilenos según comportamiento prosocial y antisocial. De este modo, el diseño de la investigación es descriptivo-correlacional-multivariante.

\section{Instrumentos}

Cuestionario de auto-reporte de Comportamiento Social Adolescente CACSA. Instrumento construido racionalmente para explorar comportamientos prosociales y antisociales, registrando frecuencias de las conductas expresadas en escala tipo Likert de 0 a 6 puntos (nunca $=0 ; 1$ ó 2 veces $=1$, varias veces $=3$, casi siempre $=5$ y siempre $=6$ ), además de consignar de modo dicotómico $(\mathrm{Si} / \mathrm{No})$ su realización durante los últimos 12 meses. El Cuestionario consta de 56 ítems, que miden tres dimensiones principales: Comportamiento Prosocial "CPRO", Comportamiento Antisocial Total "CAT" y Víctima de Abuso de Poder "VAP". Registra un continuo del comportamiento antisocial desde transgresiones en el medio escolar hasta conductas delictivas a través de 41 ítems, sin embargo, se puntúan como CAT sólo 37 ítems. De esta escala, se derivan seis subescalas: a) Delitos - D, b) Transgresión contra la Propiedad - TPRO, c) Transgresión contra las Personas - TPER, d) Comportamiento Abusivo con Pares - CAP, y e) Consumo de Alcohol y Drogas - OHD. Se aplicó adicionalmente una escala de deseabilidad social (10 ítems) - DS (Mladinic, Saiz, Díaz, Ortega, \& Oyarce, 1998), de respuesta dicotómica-V/F, con el objetivo de controlar esta variable en las respuestas de los adolescentes, dejando fuera de la muestra aquellos jóvenes con alta puntuación ( $>8$ pts.) en la escala.
Para el desarrollo de este instrumento se revisó ampliamente la literatura de inventarios en el campo del comportamiento social auto-reportado en adolescentes, seleccionándose tres instrumentos: a) Escala de conducta antisocial ABS de Allsopp y Feldman (1976) versión española (Martorell \& Silva, 1993); b) Questionnaire du MASPAQ (Le Blanc, McDuff, \& Fréchette, 1990); y c) Cuestionario de Comportamiento Prosocial de Martorell y González (1992). Estos inventarios fueron analizados en su contenido para seleccionar ítems localmente pertinentes y luego adaptarlos en lenguaje en base a criterio experto y sondeo con estudiantes secundarios. Posteriormente se creó ítems para abuso de poder entre pares, a partir de un estudio paralelo de tipo cualitativo que, mediante grupos focales, exploró el discurso juvenil sobre la violencia escolar (Arias, Guevara, \& Román, 2008). A partir de lo señalado se diseñó una versión preliminar del CACSA con 67 ítems que se sometieron a validación de contenido por jueces expertos y a una aplicación piloto con 135 estudiantes e de $1^{\circ}$ a $4^{\circ}$ medio que permitieron ajustarlo a su versión definitiva de 56 ítems (Cid, Jaramillo, Roa, \& Sanhueza, 2009).

Cuestionarios complementarios. En el marco del proyecto de investigación que sustenta este estudio (Alarcón, Vinet, Salvo, \& Pérez-Luco, 2009), se aplicó simultáneamente otros dos instrumentos de auto-reporte, a) el Cuestionario de Sucesos de Vida Estresantes CSVE (Lucio \& Durán, 2000), que cuenta con adecuados niveles de confiabilidad en muestras de adolescentes chilenos (Alarcón Banãres, Muñoz Pérez, Toirkens Niklitschek, Toledo Cárdenas, \& Venegas Villanueva, 2005); y se utilizó para establecer validez convergente con 
la subescala de problemas conductuales y b) el Inventario Clínico para adolescentes de Millon, Millon, \& Davis (1993) MACI, adaptado y validado en Chile, con adecuados niveles de confiabilidad para adolescentes escolarizados e infractores de ley. Se elige el MACI, ya que ha tenido un especial desarrollo en la psicología chilena, contando con una versión nacional (Vinet y cols., 1999) que posee buenas características psicométricas en adolescentes de población general y con trastornos psicopatológicos (Vinet \& Alarcón, 2003) y una capacidad adecuada para caracterizar a diversos grupos de adolescentes, entre ellos a adolescentes con problemas de desadaptación social (Alarcón, Vinet, \& Salvo, 2005). Además, la versión chilena del MACI posee normas propias con baremos diferenciados por sexo para adolescentes entre 13 y 19 años (Vinet \& Forns, 2008).

En la muestra de adolescentes infractores de ley se usó además un registro adicional a través de dos instrumentos de registro de comportamiento delictual, los cuales son completados por los profesionales responsables del caso en los programas de tratamiento de los jóvenes. Estos instrumentos son el YLS:CMI (Hoge \& Andrews, 1994) y la Ficha de Evaluación de Riesgos y registro de comportamiento delictivo FER (Alarcón, 2001). Estos instrumentos son interesantes porque se responden basado en conductas infractoras oficiales del adolescente, incorporando otra fuente de información, los antecedentes y los reportes de familiares acerca de su actividad delictiva.

\section{Consideraciones éticas}

Todos los adolescentes participan voluntariamente, firmando un consentimiento informado previo a responder los cuestionarios; asimismo, todas las instituciones comprometen formalmente su participación mediante documentos firmados por sus respectivos directivos.

\section{Procedimiento}

\section{Recolección de datos}

Las muestras fueron evaluadas de modo independiente: el año 2007 los estudiantes y 2008 los adolescentes infractores, sentenciados por la LRPA. El CACSA se aplicó siempre en segundo lugar, secundando el CSVE y antecediendo el MACI, con el objetivo de integrarlo en una batería de test de auto-reporte sobre vivencias adolescentes. Para la evaluación de estudiantes se seleccionó de modo intencionado los establecimientos y se hizo aplicaciones colectivas por curso. A los adolescentes infractores se les aplicó de modo individual y con formato de entrevista, privilegiando la evaluación por profesionales que trabajan en los equipos técnicos de los programas, a los que previamente se les capacitó. El tiempo de aplicación total varió entre 60 y 120 minutos tanto en la aplicación colectiva (estudiantes) como en la individual (infractores).

\section{Análisis de datos}

Los datos recogidos son analizados con el software SPSS versión 13.0, utilizando estadística descriptiva para la caracterización de las muestras, pruebas de diferencias de medias para muestras independices ( $t$ de Student y ANOVA con test post-hoc de Gabriel) y Análisis de Regresión Lineal Múltiple con pasos sucesivos para establecer los valores predictivos.

\section{Resultados}

\section{Caracterización de muestras}

La distribución del comportamiento antisocial en la muestra de estudiantes es asimétrica positiva, sin ajuste a la curva normal; y en cuanto a la relación entre comportamiento antisocial y prosocial, se observa una correlación inversa y significativa $\left(\mathrm{r}_{(1914)=}=0,063, \mathrm{p}=0,006\right)$ entre ambas manifestaciones (a mayor CAT, menor CPRO), pero el valor neto de esta asociación es muy bajo, mostrando que pese a la realización de conductas antisociales, los adolescentes que delinquen no dejan de realizar comportamientos prosociales, aunque estos sean en una tasa menor a la de sus pares normalmente escolarizados.

\section{Confiabilidad}

La consistencia interna del CACSA se estimó mediante el índice alfa de Cronbach, logrando valores superiores a 0,77 para las escalas principales de comportamiento antisocial CAT y prosocial CPRO, tanto en la muestra de estudiantes como en la de infractores y superiores a 0,65 para el resto de las subescalas. La escala CAP es la única que no logra un nivel de confiabilidad aceptable para la muestra de estudiantes (alfa de Cronbach=0,56), pero si lo hace con los infractores; (alfa de Cronbach= 0,64 ) ello se prevé producto de la alta variabilidad de comportamientos que originan al fenómeno del Bullying lo que, sin embargo, no es tema de este artículo. Resulta importante destacar que los valores alfa más altos en todas las escalas de comportamiento antisocial los obtienen de modo sistemático los infractores (Tabla 2).

La estabilidad de la medición realizada con el CACSA se estableció mediante retest, realizando una segunda aplicación del instrumento a un grupo de 42 estudiantes con una semana de tiempo intermedio a fin de correlacionar los resultados en las escalas principales CAT y CPRO. Con este procedimiento y usando el coeficiente de Pearson se pudo establecer un $\mathrm{r}_{(42)}=0,82$ para CAT y un $\mathrm{r}_{(42)}=0,89$ para CPRO, siendo ambos altamente significativas $(\mathrm{p}<0,000)$ lo que indica un alto grado de estabilidad temporal en la medición realizada.

Dados los valores de los índices de confiabilidad por consistencia interna y test-retest ya señalados, se concluye que el cuestionario mide de modo consistente y estable los comportamientos prosociales y antisociales más frecuentes en adolescentes escolarizados e infractores. 
Tabla 2

Valores Alfa y parámetros en CACSA para las muestras de estudiantes e infractores

\begin{tabular}{|c|c|c|c|c|c|c|c|c|c|c|}
\hline \multirow{2}{*}{ Escalas y Subescalas } & & \multirow{2}{*}{ Ítems } & \multicolumn{4}{|c|}{ Estudiantes } & \multicolumn{4}{|c|}{ Infractores } \\
\hline & & & Alfa & Min & Max & Media & Alfa & Min & Max & Media \\
\hline Comportamiento Prosocial & CPRO & 10 & 0,79 & & 65 & 30,0 & 0,77 & & 57 & 25,9 \\
\hline Comportamiento Antisocial Total & CAT & 37 & 0,86 & & 139 & 15,3 & 0,90 & & 130 & 46,7 \\
\hline Delitos & $\mathrm{D}$ & 13 & 0,70 & & 47 & 4,2 & 0,78 & & 39 & 11,9 \\
\hline Transgresiones contra la Propiedad & TPRO & 10 & 0,65 & & 34 & 2,7 & 0,75 & & 42 & 12,1 \\
\hline Transgresiones contra las Personas & TPER & 14 & 0,72 & & 29 & 4,8 & 0,76 & & 48 & 10,8 \\
\hline Comportamiento Abusivo con Pares & CAP & 6 & 0,56 & & 22 & 1,8 & 0,64 & & 27 & 4,0 \\
\hline Alcohol y Drogas & OHD & 7 & 0,74 & & 35 & 2,7 & 0,80 & & 51 & 15,1 \\
\hline Transgresiones Sociales Leves & TSL & 9 & 0,73 & & 59 & 7,4 & 0,74 & & 43 & 15,6 \\
\hline Víctima de Abuso de Poder & VAP & 5 & 0,51 & & 21 & 2,4 & 0,55 & & 19 & 2,2 \\
\hline Deseabilidad Social & DS & 10 & 0,57 & 4 & 10 & 6,7 & 0,27 & 5 & 10 & 6,9 \\
\hline
\end{tabular}

\section{Validez discriminante y convergente}

Para establecer empíricamente la validez del auto-reporte de comportamiento registrado por el CACSA, se analizó su capacidad para discriminar de tres modos distintos: a) entre respuestas de dos grupos de adolescentes varones con antecedentes de comportamiento antisocial divergente; una muestra de adolescentes escolarizados $(\mathrm{N}=901)$, en los que se presume un comportamiento habitual socialmente adaptativo, y otra de infractores de ley que cumplen condena en algún programa de intervención de SENAME (N=262), obteniéndose diferencias altamente significativas $(\mathrm{p}=0,000)$ en todas las subescalas de CAT con mayores valores para los infractores (Tabla 3 y Figura 1); b) Comparación entre hombres $(\mathrm{N}=901)$ y mujeres $(\mathrm{N}=1.013)$, pues se asume que las diferencias de género en las tasas de delincuencia son resultantes de diferencias generales de comportamiento por sexo (Cooper, 2002), los resultados muestran valores significativamente más altos $(\mathrm{p}=0,000)$ en los hombres en todas las dimensiones de CAT e inferiores en CPRO, resultando teóricamente coherente (ver Tabla 3 y Figura 1); c) Comparando las respuestas de los infractores entre sí, subdivididos de acuerdo a intensidad del compromiso delictual e incluyendo al grupo de estudiantes que auto-reporta alto puntaje en el CAT, esto implica comparar cuatro grupos formados en base a criterios externos de compromiso delictual: Auto-reporte, Inicio, Transición y Persistencia (caracterización en descripción de participantes), los resultados muestran diferencias altamente significativas entre los cuatro grupos $(\mathrm{p}=0,000)$, observándose que los valores más altos los señalan sistemáticamente los persistentes y los más bajos los de inicio, lo que es teóricamente consistente y se ajusta a lo esperado. La comparación de adolescentes infractores de ley con un grupo que no ha sido detenido o judicializado y auto-reporta alto comportamiento antisocial muestra valores muy similares al grupo de transición delictiva e incluso mayor al grupo persistente en transgresiones a las personas (Tabla 4 y Figura 2).

Tabla 3

Tabla comparativa de puntuaciones en CACSA según muestra y sexo

\begin{tabular}{|c|c|c|c|c|c|c|}
\hline \multirow{3}{*}{ Variables CACSA } & \multicolumn{6}{|c|}{ Diferencias entre las muestras } \\
\hline & \multicolumn{2}{|c|}{$\begin{array}{c}\text { Estudiantes } \\
(\mathbf{N}=901)\end{array}$} & \multicolumn{2}{|c|}{$\begin{array}{c}\text { Infractores } \\
(\mathbf{N}=\mathbf{2 6 2})\end{array}$} & \multicolumn{2}{|c|}{ U de Mann-Whitney } \\
\hline & Media & Mediana & Media & Mediana & $\mathbf{U}$ & $\mathbf{p}$ \\
\hline Comportamiento Prosocial & 30,0 & 30 & 25,9 & 25 & 93,948 & 0,0000 \\
\hline Comportamiento Antisocial Total & 15,3 & 12 & 46,7 & 45 & 31,659 & 0,0000 \\
\hline Delitos & 4,2 & 3 & 15,1 & 14 & 36,312 & 0,0000 \\
\hline Trasgresiones contra la Propiedad & 2,7 & 1 & 11,9 & 11 & 33,079 & 0,0000 \\
\hline Trasgresiones contra las Personas & 4,8 & 3 & 10,8 & 9 & 62,546 & 0,0000 \\
\hline Comportamiento Abusivo con Pares & 1,8 & 1 & 4,0 & 3 & 77,809 & 0,0000 \\
\hline Alcohol y Drogas & 2,7 & 1 & 12,1 & 12 & 31,518 & 0,0000 \\
\hline Trasgresiones Sociales Leves & 7,4 & 6 & 15,6 & 14 & 51,197 & 0,0000 \\
\hline Víctima de Abuso de Poder & 2,4 & 2 & 2,2 & 1 & 106,311 & 0,0124 \\
\hline
\end{tabular}


Tabla 3

(continuación de la página anterior)

\begin{tabular}{|c|c|c|c|c|c|c|}
\hline \multirow{3}{*}{ Variables CACSA } & \multicolumn{6}{|c|}{ Diferencias según sexo } \\
\hline & \multicolumn{2}{|c|}{ Hombre } & \multicolumn{2}{|c|}{ Mujer } & \multicolumn{2}{|c|}{ U de Mann-Whitney } \\
\hline & Media & Mediana & Media & Mediana & $\mathbf{U}$ & $\mathbf{p}$ \\
\hline Comportamiento Prosocial & 30,0 & 30 & 35,3 & 36 & 333,330 & 0,0000 \\
\hline Comportamiento Antisocial Total & 15,3 & 12 & 11,6 & 8 & 374,698 & 0,0000 \\
\hline Delitos & 4,2 & 3 & 1,8 & 1 & 271,261 & 0,0000 \\
\hline Trasgresiones contra la Propiedad & 2,7 & 1 & 1,7 & 1 & 396,184 & 0,0000 \\
\hline Trasgresiones contra las Personas & 4,8 & 3 & 3,3 & 2 & 370,933 & 0,0000 \\
\hline Comportamiento Abusivo con Pares & 1,8 & 1 & 1,3 & - & 402,025 & 0,0000 \\
\hline Alcohol y Drogas & 2,7 & 1 & 2,4 & - & 430,367 & 0,0217 \\
\hline Trasgresiones Sociales Leves & 7,4 & 6 & 6,7 & 5 & 425,184 & 0,0096 \\
\hline Víctima de Abuso de Poder & 2,4 & 2 & 2,7 & 2 & 425,403 & 0,0092 \\
\hline
\end{tabular}

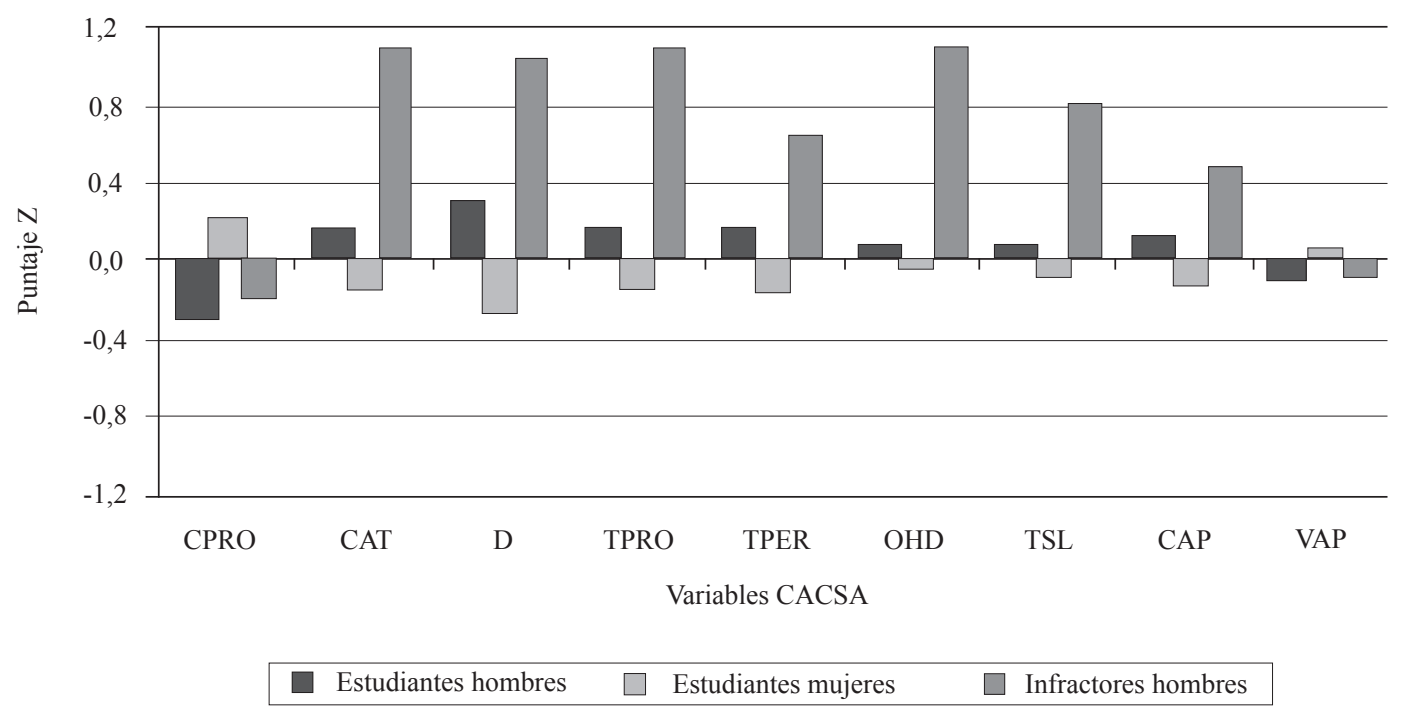

Figura 1. Comportamiento prosocial y antisocial de estudiantes secundarios e infractores de ley.

Tabla 4

Tabla comparativa de puntuaciones en CACSA de las cuatro muestras estudiadas

\begin{tabular}{|c|c|c|c|c|c|c|c|}
\hline & \multicolumn{4}{|c|}{ Medias } & \multicolumn{3}{|c|}{ ANOVA } \\
\hline & Autoreporte & Inicio & Transición & Persistencia & gl & $\mathbf{F}$ & Sig. \\
\hline Comportamiento Prosocial & 30,5 & 26,5 & 26,5 & 24,9 & 384 & 4,51 & 0,0040 \\
\hline Comportamiento Antisocial Total & 41,4 & 33,4 & 45,9 & 60,7 & 384 & 24,61 & 0,0000 \\
\hline Delitos & 13,0 & 9,5 & 16,5 & 19,6 & 384 & 23,84 & 0,0000 \\
\hline Trasgresiones contra la Propiedad & 8,5 & 7,6 & 12,6 & 15,7 & 384 & 26,74 & 0,0000 \\
\hline Trasgresiones contra las Personas & 13,8 & 8,3 & 10,8 & 13,3 & 384 & 7,92 & 0,0000 \\
\hline Comportamiento Abusivo con Pares & 5,2 & 2,7 & 3,9 & 5,4 & 384 & 7,59 & 0,0001 \\
\hline Consumo de Alcohol y Drogas & 8,5 & 8,1 & 10,7 & 17,2 & 384 & 37,81 & 0,0000 \\
\hline Trasgresiones Sociales Leves & 16,8 & 13,0 & 14,8 & 18,7 & 384 & 8,99 & 0,0000 \\
\hline Víctima de Abuso de Poder & 4,0 & 1,6 & 2,4 & 2,7 & 384 & 10,08 & 0,0000 \\
\hline
\end{tabular}

Se resaltan con negritas las diferencias estadísticamente significativas según la prueba Post-Hoc de Gabriel 
Alarcón Bañares, P., Pérez-Luco, R., Salvo, S., Roa, G., Jaramillo, K., \& Sanhueza, C. (2010). Validação de CACSA.

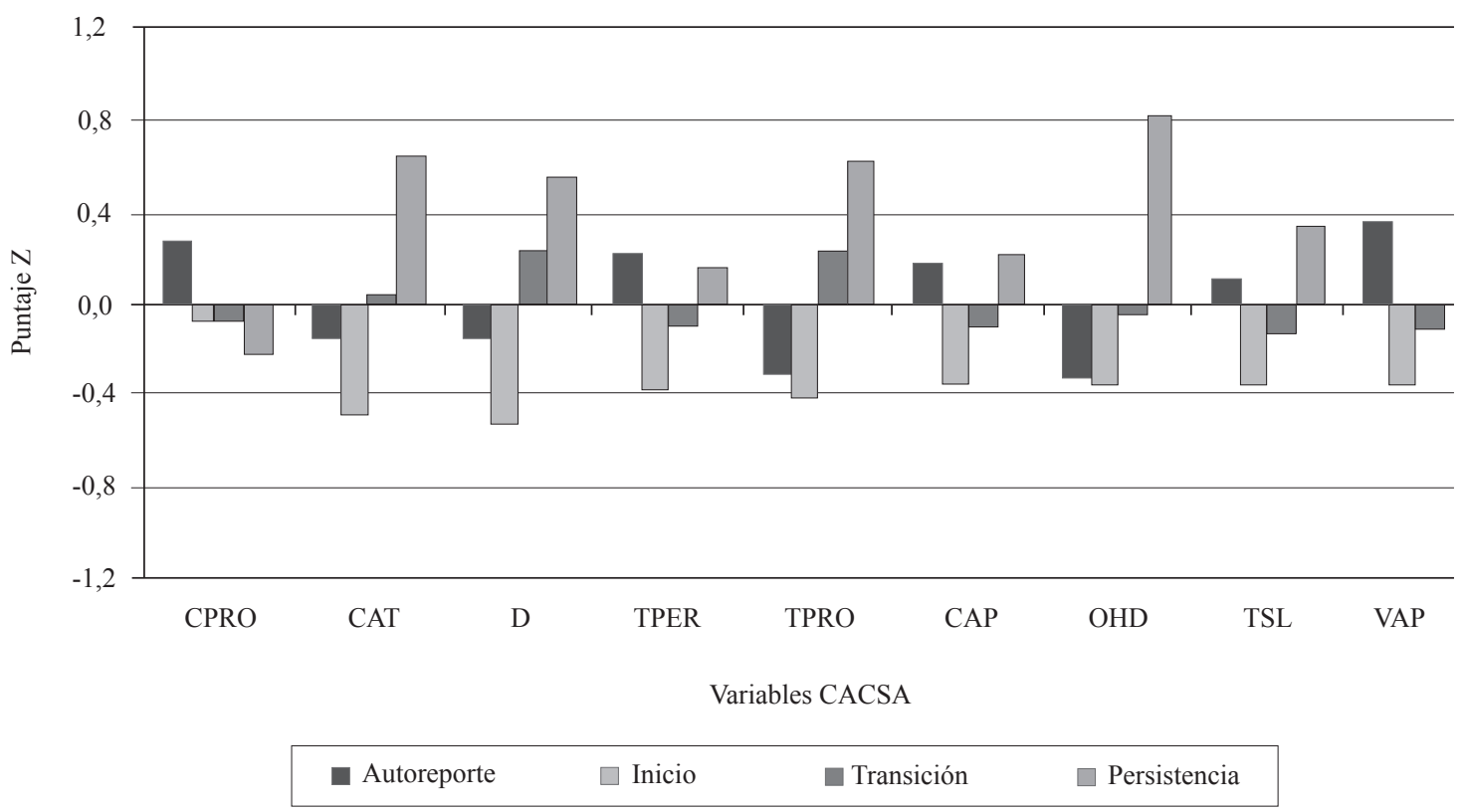

Figura 2. Comportamiento prosocial y antisocial en las cuatro muestras de varones.

Para determinar empíricamente la validez convergente del CACSA se exploró con todos los participantes (1914 estudiantes hombres y mujeres y 264 infractores varones) el nivel de asociación del índice de comportamiento antisocial (CAT) con las escalas predisposición delictual y trasgresor del MACI y se establece la asociación con el factor problemas de conducta del CSVE, estas sub pruebas responden a constructos teóricamente relacionados. Se observa que para la muestra de estudiantes (E) y la de infractores (I), el puntaje CAT correlaciona positiva y significativamente con predisposición delictual $\left(\mathrm{r}_{\mathrm{E}}=0,46: \mathrm{p}=, 000 ; \mathrm{r}_{\mathrm{I}}=0,37: \mathrm{p}=, 000\right)$, transgresor $\left(r_{E}=0,59: p=, 000 ; r_{I}=0,54: p=, 000\right)$ y problemas de conducta $\left(r_{E}=0,63: p=0,000 ; r_{I}=0,60: p=, 000\right)$. Además se exploró, para cada uno de los participantes de la muestra de infractores, la relación entre puntaje CAT y puntuaciones de comportamiento delictual observadas por educadores y profesionales mediante registro de antecedentes delictuales puntuado en factor 1 en el instrumentos de Riesgo YLS:CMI y con el índice de escalada delictual IEDS de la ficha FER, encontrando correlación positiva y significativa con los índices de riesgo de reincidencia del YLS-CMI y de escalada de desadaptación social IEDS $\left(r_{\text {IRBC }}=0,55: p=, 0000 ; r_{\text {IEDS }}=0,37\right.$ : $\mathrm{p}=, 0000)$. Todos los resultados de éste análisis dan cuenta de alta asociación entre constructos e índices teóricamente similares mostrando convergencia de mediciones con indicadores ya validados para la población adolescente chilena (Alarcón, 2001; Chesta, 2009).

Las comparaciones descritas muestran diferencias y asociaciones estadísticamente muy significativas en todas las subescalas y en los diferentes escenarios de comparación, lo que permite afirmar que el CACSA discrimina entre adolescentes con y sin delitos oficiales y entre grupos que teóricamente se espera presenten distinto nivel de comportamiento antisocial y prosocial.

\section{Discusión y Conclusiones}

El presente estudio permite concluir que el CACSA es un buen instrumento para registro y medición de auto-reporte de comportamientos antisociales en adolescentes chilenos, secundariamente detecta conductas prosociales y su relación con lo antisocial. Se demuestra que el instrumento cuenta con una buena confiabilidad por consistencia interna y estabilidad de la medida y su validez interna (convergente) y externa (discriminante), permite sustentar adecuadas propiedades psicométricas.

El que la tasa de comportamiento antisocial se distribuya de modo asimétrico positivo en la muestra de estudiantes resulta teóricamente coherente, pues la mayoría de los adolescentes muestran bajos niveles de comportamientos antisociales y no más del $13 \%$ presenta alto reporte de conductas delictivas, en cambio los comportamientos prosociales revelan una distribución más cercana a la curva normal y las frecuencias se distribuyen de modo más homogéneo en la muestra, siendo más alta la tasa de comportamientos prosociales que antisociales y más frecuentes en mujeres que en hombres, lo que demuestra que los adolescentes, por regla general, realizan más comportamientos prosociales que antisociales. Este es un hallazgo interesante que propone una relación compleja entre lo prosocial y antisocial en la adolescencia y viene a cuestionar al menos en un subgrupo de adolescentes, una clara relación inversamente proporcional, 
entre lo prosocial y lo antisocial descrito por López y cols. (2002) y por Mestre y cols. (2002), ya que un grupo de adolescentes chilenos delinque de modo frecuente pero a su vez realiza conductas prosociales con sus pares, "reapareciendo" el mito urbano asociado a la representación de "Robin Hood".

En cuanto al comportamiento antisocial en el grupo de infractores el auto-reporte permite detectar una brecha importante en el abuso de alcohol y drogas, donde quienes delinquen muestran niveles de consumo significativamente más altos que los que no lo hacen (Tabla 3). Esto reafirma resultados anteriores (CONACE, 2008; Garrido Martín, Herrero Alonso, \& Masip Pallejá, 2002; Lepre \& Martins, 2009) que muestran la alta asociación entre conductas antisociales y consumen alcohol y drogas.

Las diferencias de género son confirmadas en este estudio tanto para lo prosocial como lo antisocial. Estos hallazgos coinciden con los antecedentes reportados por investigadores españoles (Mestre y cols., 2007), empero, la relación encontrada se acerca a 2:1 mostrando que la tasa de mujeres con alto comportamiento antisocial difiere bastante del 3:1 ó 4:1 reportado por Rutter y cols. (2000).

Los resultados obtenidos en la escala de víctima de abuso de poder VAP, invitan a revisar esta subescala ya que está débilmente representada en cinco ítemes y sus índices de confiabilidad son bajos. Es una dimensión que requiere ser mejorada ya que el instrumento no logra medir un fenómeno de alta complejidad como éste, donde las variables de clima escolar, pautas de violencia familiar y educacional no son abordadas en el instrumento estudiado. Por otra parte, hay desafíos pendientes en el tema de adolescentes mujeres infractoras de ley, no se contrasta con esta población, por tanto, es difícil describir la relación entre lo prosocial y lo antisocial en ellas.

En síntesis se aporta con un instrumento nuevo y sensible para evaluar a los adolescente chilenos, permite conocer la aceptación y frecuencia de comportamientos sociales considerados aceptables, permite detectar por auto-reporte que las medias obtenidas por los adolescentes que enfrentan al sistema judicial no difieren significativamente de aquellas conductas de adolescentes que se mantienen adheridos al medio escolar, acercándose a un grupo de transición delictiva. En conclusión, se puede afirmar que el CACSA mide efectivamente comportamientos antisocial y prosocial, a través de este estudio discrimina de modo certero entre quienes transgreden la ley o participan del mundo delictivo y quienes expresan comportamientos socialmente adaptativos y prosociales. Los datos apoyan los hallazgos de que la técnica del auto-reporte es una metodología prometedora para explorar, cuantificar y describir el comportamiento transgresor adolescente.

\section{Referencias}

Alarcón Banãres, A., Muñoz Pérez, E., Toirkens Niklitschek, J., Toledo Cárdenas, L., \& Venegas Villanueva, M. (2005). Adaptación del inventario de sucesos de vida (versión para adolescentes) de Lucio y Durán (2000) con jóvenes en la Ciudad de Temuco. Trabajo de realización del curso no publicado, Universidad de La Frontera, Temuco.

Alarcón, P. (2001). Evaluación psicológica de adolescentes con desadaptación social: Un estudio através del MACI e indicadores de riesgo en el sur de Chile. Tesis de master no publicada, Universidad de Salamanca, Salamanca.

Alarcón, P., Vinet, E., \& Salvo, S. (2005). Estilos de personalidad y desadaptación social durante la adolescencia. Psykhe, 14(1), 3-16.

Alarcón, P., Vinet, E., Salvo, S., \& Pérez-Luco, R. (2009). Caracterización y evaluación multidimensional de adolescentes con desadaptación social (Informe Final Fondecyt No 1070397). Temuco: Universidad de La Frontera.

Allsopp, B., \& Feldman, R. (1976). Personality and anti-social behaviour in schoolboys: Item analysis of questionnaire measures. British Journal of Criminology, 16, 337-351.

Arias, A., Guevara, L., \& Román, C. (2008). Caracterización del comportamiento social y autorreporte de Bullying en adolescentes secundarios de cinco ciudades del sur de Chile. Trabajo de realización del curso no publicado, Universidad de La Frontera, Temuco.

Bringas, C., Herrero, F., Cuesta, R., \& Rodríguez, F. (2006). La conducta antisocial en adolescentes no conflictivos: Adaptación del Inventario de Conductas Antisociales (ICA). Revista Electrónica de Metodología Aplicada, 11(2), 1-10. Recuperado en 10 diciembro 2009, de http:// www.psico.uniovi.es/rema/v11n2/antisoc.pdf

Calvo, A., González, R., \& Martorell, M. (2001). Variables relacionadas con la conducta prosocial en la infancia y adolescencia: Personalidad, autoconcepto y género. Infancia y Aprendizaje, 24, 95-111.

Caprara, G. V., Steca, P., Zelli, A., \& Capanna, C. (2005). A new scale for measuring adults' prosocialness. European Journal of Psychological Assessment, 21, 77-89.

Cid, M., Jaramillo, K., Roa, G., \& Sanhueza, C. (2009). Cuestionario de auto-reporte de comportamiento social adolescente (CACSA): Diseño y validación preliminar. Trabajo de realización del curso no publicada, Universidad de La Frontera, Temuco.

Consejo Nacional para el Control de Estupefacientes. (2003). Quinto estudio nacional de drogas en población escolar de chile, 2003: $8^{\circ}$ Básico a $4^{\circ}$ Medio. Informe final. Santiago: CONACE.

Consejo Nacional para el Control de Estupefacientes. (2007). Séptimo estudio nacional de drogas en población escolar de chile, 2007: $8^{\circ}$ Básico a $4^{\circ}$ Medio. Informe de principales resultados. Santiago: CONACE. 
Consejo Nacional para el Control de Estupefacientes. (2008). Taller práctico de evaluación clínica, detección, sospecha y confirmación diagnostica del consumo problemático de drogas en adolescentes [presentación de diapositivas]. Santiago: CONACE.

Cooper Mayr, D. (2002). Criminología y delincuencia femenina en Chile. Santiago: LOM.

Chesta, S. (2009). Características psicométricas del inventario de riesgos y necesidades vinculados con factores criminogénicos: IRBC. Tesis de master no publicada, Universidad de La Frontera, Temuco.

Dionne, J., \& Cournoyer, L. (2006). Trajectories délinquantes: La réadaptation est possible... a certains conditions. Prisme, 45, 206-217.

Farrington, D. P. (1996). The explanation and prevention of youthful offending. In P. Cordelia \& L. Siegel (Eds.), Readings in contemporary criminological theory (pp. 257-272). Boston: Northeastern University Press.

Farrington, D. P., Jolliffe, D., Hawkins, J. D., Catalano, R. F., Hill, K. G., \& Kosterman, R. (2003). Comparing delinquency careers in court records and self-reports. Criminology, 41, 933-958.

Fréchette, M., \& Le Blanc, M. (1998). Délinquances et délinquants (8ème ed.). Montréal: Gaêtan Morin.

Garaigordobil, M., Maganto, C., Perez, J. I., \& Sansinenea, E. (2009). Gender differences in socioemotional factors during adolescence and effects of a violence prevention program. Journal of Adolescent Health, 44, 468-477.

García, M., \& Madriaza, P. (2005). Sentido y sinsentido de la violencia escolar: Análisis cualitativo del discurso de estudiantes chilenos. Psykhe, 14(1), 165-180.

Garrido Martín, E., Herrero Alonso, C., \& Masip Pallejá, J. (2002). Autoeficacia y delincuencia. Psicothema, 14(Supl. 1), 63-71.

Garrido, V., Stangeland, P., \& Redondo, S. (1999). Principios de criminología. Valencia: Tirant lo Blanch.

González, R., Martorell, C., Casulla, M. M., \& Calvo, A. J. (1998). Comparación entre estudiantes argentinos y españoles. Acta Psiquiátrica y Psicológica, 44, 257-267.

Gracia, E., \& Herrero, J. (2006). La comunidad como fuente de apoyo social: Evaluación e implicaciones en los ámbitos individual y comunitario. Revista Latinoamericana de Psicología, 38, 327-342.

Hein, A., \& Barrientos, G. (2004). Violencia y delincuencia juvenil: Comportamientos auto reportados y factores asociados. Santiago: Fundación Paz Ciudadana.

Hoge, R. D., \& Andrews, D. A. (1994). The youth level of service: Case managment. inventory and manual. Ottawa: Carlenton University.

Huizinga, D., \& Elliott, D. S. (1986). Reassessing the reliability and validity of self-report delinquency measures. Journal of Quantitative Criminology, 2, 293-327.
Inglés, C. J., Benavides, G., Redondo, J., García-Fernández, J. M., Ruiz-Esteban, C., Estévez, C., \& Huéscar, E. (2009). Conducta prosocial y rendimiento académico en estudiantes españoles de educación secundaria obligatoria. Anales de Psicología, 25, 93-101.

Junge-Tas, J., Terlouw, G. J., \& Klein, M. W. (1994). Delinquent behavior among young people in the western world. Amsterdam: Kugler.

Le Blanc, M., McDuff, P., \& Fréchette, M. (1990). MASPAQ: Measures de la adaptation social et personnelle pour les adolescenters québécois. Montréal: Université de Montreal.

Lepre, R. M., \& Martins, R. A. (2009). Raciocínio moral e uso abusivo de bebidas alcoólicas por adolescentes. Paidéia (Ribeirão Preto), 19, 39-45.

López Latorre, M. J., Garrido Genovés, V., Rodríguez Díaz, F. J., \& Paíno Quesada, S. G. (2002). Jóvenes y competencia social: Un programa de intervención. Psicothema, 14(Supl. 1), 156-163.

Lucio, E., \& Durán, C. (2000). Sucesos de vida, versión para adolescentes. México: Manual Moderno.

Maluf, N. A. (2002, Mayo). Las subjetividades juveniles en sociedades en riesgo: Un análisis en contextos de globalización y modernización. Conferencia dictada en Seminario Globalización y Antiglobalización en Europa y América Latina, Lleida-Barcelona, España.

Martorell, M. C., \& Gonzalez, E. (1992, Septiembre). Cuestionario de Conductaprosocial (CCP) y Cuestionario de Conducta Antisocial (CCA). Conferencia dictada en 4. Congreso de Evaluación Psicológica, Santiago de Complostela, España.

Martorell, M. C., \& Silva, F. (1993). ASB Escala de conducta antisocial. In F. Silva \& M. C. Martorell(Eds.), Evaluación infanto-juvenil (pp. 83-110). Madrid: MEPSA.

Marzocchi, G. M., Capron, C., Di Pietro, M., Duran Tauleria, E., Duyme, M., Frigerio, A., Gaspar, M. F., Hamilton, H., Pithón, G., Simões, A., \& Thérond, C. (2004). The use of Strengths and Difficulties Questionnaire (SDQ) in Southern European countries. European Child and Adolescent Psychiatry, 13(Suppl. 2), 1140-1146.

Mestre Escrivá, M., Samper García, P., \& Frías Navarro, M. (2002). Procesos cognitivos y emocionales predictores de la conducta prosocial y agresiva: Empatía como factor modulador. Psicothema, 14, 227-232.

Mestre, V., Samper, P., Nacher, M. J, Tur, A., \& Cortés, M. T. (2006). Psychological processes and family variables as prosocial behavior predictors in a sample of Spanish adolescents. Psychological Reports, 98, 30-36.

Mestre, M. V., Tur, A., Samper, P., Nacher, M. J., \& Cortés, M. T. (2007). Estilos de crianza en la adolescencia y su relación con el comportamiento prosocial. Revista Latinoamericana de Psicología, 39, 211-225.

Millon, T., Millon, C., \& Davis, R. (1993). Manual of Millon Adolescent Clinical Inventory. Minneapolis: National Computer Systems. 
Mladinic, A., Saiz, J. L., Díaz, M., Ortega, A., \& Oyarce, P. (1998). Sexismo ambivalente en estudiantes universitarios chilenos: Teoría, medición y diferencias de género. Revista de Psicología Social y Personalidad, 14, 1-14.

Moffitt, T. E. (1993). Adolescence-limited and lifecourse-persistent antisocial behavior: A developmental taxonomy. Psychological Review, 100, 674-701.

Olweus, D. (1993). Bullying at school: What we know and what we can do. Oxford: Blackwell.

Ortega Ruiz, R., \& Del Rey Alamillo, R. (2005). Violencia interpersonal y bullying en la escuela. Conferencia dictada no Congreso Ser Adolescente Hoy, Madrid, España.

Roa, M. L., Del Barrio, M. V., \& Carrasco, M. A. (2004). Comparación de la agresión infantil en dos grupos culturales. Revista de Psicología, 22, 30-43.

Rutter, M., \& Giller, H. (1985). Delincuencia juvenil. Barcelona: Martínez Roca.

Rutter, M., Giller, H., \& Hagell, A. (2000). La conducta antisocial de los jóvenes. Madrid: Cambridge University Press.

Scheaffer, R. L., Mendenhall, W., \& Ott, L. (1987). Elementos de muestreo. México: Grupo Editorial Iberoamérica.

Seisdedos, N. (1988). A-D cuestionario de conductas antisociales-delictivas. Madrid: TEA.

Serrano, G., El-Astal, S., \& Faro, F. (2004). La adolescencia en España, Palestina y Portugal: Análisis comparativo. Psicothema, 16, 468-475.

Tur, A. M., Mestre, M. V., \& Barrio, M. V. (2004). Factores moduladores de la conducta agresiva y prosocial. El efecto de los hábitos de crianza en la conducta del adolescente. Ansiedad y Estrés, 10, 75-88.

UNICEF. (2004). Informe anual de actividades: Chile, 2004. Recuperado en 10 diciembro 2009, de http:/www.unicef. cl/archivos_documento/128/INFORME\%202004.pdf

Vinet, E., \& Alarcón, P. (2003). El Inventario Clínico para Adolescentes de Millon (MACI) en la evaluación de adolescentes chilenos. Psykhe, 12, 39-55.

Vinet, E., Brio, C., Correa, P., Díaz, P., Diez, M., Echeverría, M., Salazar, D., \& Vargas, A. (1999). MACI, traducción y adaptación chilena para usos exclusivo en investigación (Proyecto de Investigación DIDUFRO 9966). Temuco: Universidad de La Frontera.

Vinet, E., \& Forns i Santacana, M. (2008). Normas chilenas para el MACI: Una integración de criterios categoriales y dimensionales. Terapia Psicológica, 26, 151-163.

Werth, F. \& Sepúlveda M. (2003) Delincuencia juvenil en Chile: Tendencias y desafios. Documento presentado en el seminario: Gobierno local y prevención en seguridad ciudadana. Santiago: Fundación Paz Ciudadana. Recuperado noviembre 2009 en http://www. pazciudadana.cl/docs/pub_20091114185009.pdf
Paula Alarcón Bañares es académica y investigadora del Departamento de Psicología, Facultad de Educación, Universidad de la Frontera, Temuco-Chile.

Ricardo Pérez-Luco es académico y investigador del Departamento de Psicología, Facultad de Educación, Universidad de la Frontera. Temuco-Chile.

Sonia Salvo es académica y investigadora del Departamento de Matemáticas y Estadística, Facultad de Ingeniería, Universidad de la Frontera, Temuco-Chile.

Gloria Roa es tesista de Licenciatura en Psicología del Departamento de Psicología, Facultad de Educación, Universidad de la Frontera, Temuco-Chile.

Katherine Jaramillo es tesista de Licenciatura en Psicología del Departamento de Psicología, Facultad de Educación, Universidad de la Frontera, Temuco-Chile.

Carla Sanhueza es tesista de Licenciatura en Psicología del Departamento de Psicología, Facultad de Educación, Universidad de la Frontera, Temuco-Chile.

Recebido: 21/05/2009

$1^{a}$ revisão: $15 / 09 / 2009$

$2^{a}$ revisão: $24 / 02 / 2010$

Aceite final: 30/03/2010 